FedUni ResearchOnline

http://researchonline.federation.edu.au

This is the published version of the following article:

Gao, D. (2016) On unified modeling, theory, and method for solving multi-scale global optimization problems. AIP Conference Proceedings, 1776, 1-8.

Which has been published in final form at:

http://doi.org/10.1063/1.4965311 


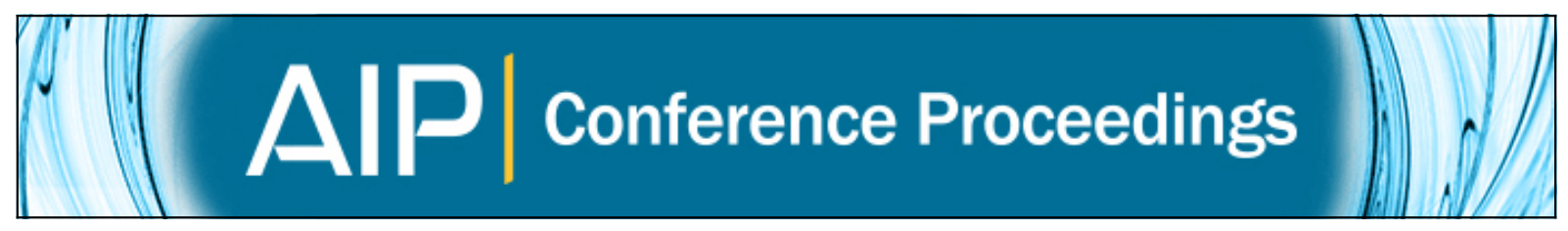

On unified modeling, theory, and method for solving multi-scale global optimization problems

David Yang Gao

Citation: AIP Conference Proceedings 1776, 020005 (2016); doi: 10.1063/1.4965311

View online: http://dx.doi.org/10.1063/1.4965311

View Table of Contents: http://scitation.aip.org/content/aip/proceeding/aipcp/1776?ver=pdfcov

Published by the AIP Publishing

\section{Articles you may be interested in}

Solving global optimization problems on GPU cluster

AIP Conf. Proc. 1738, 400006 (2016); 10.1063/1.4952194

Vector direction of filled function method on solving unconstrained global optimization problem

AIP Conf. Proc. 1707, 050011 (2016); 10.1063/1.4940843

Multi-scale modelling of uranyl chloride solutions

J. Chem. Phys. 142, 024501 (2015); 10.1063/1.4905008

Multi-scale modelling of granular avalanches

AIP Conf. Proc. 1542, 1250 (2013); 10.1063/1.4812165

Efficient Computational Strategies for Solving Global Optimization Problems

Comput. Sci. Eng. 12, 74 (2010); 10.1109/MCSE.2010.85 


\title{
On Unified Modeling, Theory, and Method for Solving Multi-Scale Global Optimization Problems
}

\author{
David Yang Gao \\ Faculty of Science and Technology, Federation University, Mt Helen, Victoria 3353, Australia \\ d.gao@federation.edu.au
}

\begin{abstract}
A unified model is proposed for general optimization problems in multi-scale complex systems. Based on this model and necessary assumptions in physics, the canonical duality theory is presented in a precise way to include traditional duality theories and popular methods as special applications. Two conjectures on NP-hardness are proposed, which should play important roles for correctly understanding and efficiently solving challenging real-world problems. Applications are illustrated for both nonconvex continuous optimization and mixed integer nonlinear programming.
\end{abstract}

\section{INTRODUCTION AND MOTIVATION}

General problems in mathematical optimization are usually formulated in the following form

$$
\min f(\mathbf{x}), \quad \text { s.t. } \mathbf{g}(\mathbf{x}) \leq 0,
$$

where the unknown $\mathbf{x} \in \mathbb{R}^{n}$ is a vector, $f(\mathbf{x}): \mathbb{R}^{n} \rightarrow \mathbb{R}$ is the so-called "objective" function" ${ }^{1}, \mathbf{g}(\mathbf{x})=\left\{g_{j}(\mathbf{x})\right\}: \mathbb{R}^{n} \rightarrow \mathbb{R}^{m}$ is a vector-valued constraint function. It must be emphasized that, different from the basic concept of objectivity in continuum physics and nonlinear analysis, the objective function used extensively in optimization literature is allowed to be any arbitrarily given function, even the linear function. Therefore, this mathematical problem is artificial. Although it enables one to "model" a very wide range of problems, it comes at a price: many global optimization problems are considered to be NP-hard. Without detailed information on these arbitrarily given functions, it is impossible to have a powerful theory for solving the general nonconvex problem (1).

Canonical duality-triality is a newly developed and continuously improved methodological theory. This theory was developed from Gao and Strang's original work on nonconvex variational/boundary value problems in large deformation mechanics [3]. It was shown in Gao's book [2] and in the recent articles [14] that both the (external) penalty and Lagrange multiplier methods are special applications of the canonical duality theory in convex optimization. It is now understood that this theory reveals an intrinsic multi-scale duality pattern in complex systems, many popular theories and methods in nonconvex analysis, global optimization, and computational science can be unified within the framework of the canonical duality-triality theory [10]. Indeed, it is easy to show that the popular semi-definite programming (SDP) methods in global optimization and the half-quadratic regularization are naturally included in the framework of the canonical duality theory $[12,16]$. This paper presents the canonical duality theory in a systematical way from a unified modeling, basic assumptions to the theory, method, and applications.

\section{MULTI-SCALE MODELING AND PROPERLY POSED PROBLEMS}

General optimization problem in a multi-scale system should be in the following form

$$
\left(\mathcal{P}_{0}\right): \min \left\{\Pi(\chi)=W(\mathbf{D} \chi)-F(\chi) \mid \chi \in \mathcal{X}_{c}\right\}
$$

\footnotetext{
${ }^{1}$ This terminology is used mainly in English literature. The function $f(\mathbf{x})$ is called the target function in Chinese and Japanese literature.
} 
In this paper, we restrict our discussion in finite-dimensional space $\mathcal{X}$. Its element $\chi \in \mathcal{X}_{a} \subset \mathcal{X}$ could be a vector, a matrix, or a tensor. $\mathbf{D}: \mathcal{X}_{a} \rightarrow \mathcal{W}$ is a linear operator which assigns each $\chi$ to an internal variable $\mathbf{w}=\mathbf{D} \chi$ in a generalized matrix space $\mathcal{W}$ equipped with a natural norm $\|\mathbf{w}\|$. The real-valued function $F: \mathcal{X}_{a} \rightarrow \mathbb{R}$ is the so-called external energy, dually, $W: W_{a} \subset \mathcal{W} \rightarrow \mathbb{R}$ is the internal energy. In $\mathcal{X}_{a}$ the geometrical constraints are pre-described for each particular problem; while $\mathcal{W}_{a}$ contains certain physical (constitutive) constraints of the system. The feasible set $\mathcal{X}_{c}=\left\{\chi \in \mathcal{X}_{a} \mid \mathbf{D} \chi \in \mathcal{W}_{a}\right\}$.

Objectivity is a basic concept in mathematical modeling and nonlinear analysis [4]. Let $\mathcal{R}$ be a special orthogonal group, i.e. $\mathbf{R} \in \mathcal{R}$ if and only if $\mathbf{R}^{T}=\mathbf{R}^{-1}$ and $\operatorname{det} \mathbf{R}=1$. A mathematical definition was given in Gao's book [2].

Definition 1 (Objectivity) $\quad A$ set $\mathcal{W}_{a}$ is said to be objective if $\mathbf{R w} \in \mathcal{W}_{a} \quad \forall \mathbf{w} \in \mathcal{W}_{a}, \forall \mathbf{R} \in \mathcal{R}$. A real-valued function $W: W_{a} \rightarrow \mathbb{R}$ is said to be objective if

$$
W(\mathbf{R w})=W(\mathbf{w}) \quad \forall \mathbf{w} \in \mathcal{W}_{a}, \forall \mathbf{R} \in \mathcal{R} .
$$

Geometrically speaking, an objective function is rotational symmetry, which should be a $\mathrm{SO}(n)$-invariant in $n$ dimensional Euclidean space. Physically, an objective function doesn't depend on observers. It was emphasized by P. Ciarlet that the objectivity is not an assumption, but an axiom [4]. Therefore, the objectivity is essential for real-world mathematical models. In mathematical physics, the internal energy $W(\mathbf{w})$ must be an objective function such that its variation (Gâteau derivative) $\sigma=\partial W(\mathbf{w})$ is the so-called constitutive duality law, which depends only on the intrinsic property of the system. Dually, the external energy $F(\chi)$ can be called the subjective function, which depends on each problem such that its variation leads to the action-reaction duality law: $\mathbf{f}=\partial F(\chi) \in \mathcal{X}_{a}^{*}$. Let $\mathcal{X}_{a}^{*} \subset \mathcal{X}^{*}$ be an input space such that for any given input $\mathbf{f} \in \mathcal{X}_{a}^{*}$ the subjective function $\langle\chi, \mathbf{f}\rangle \geq 0 \quad \forall \chi \in \mathcal{X}_{a}$. A system is conservative if the action is independent of the reaction. Therefore, the subjective function must be linear on its domain $\mathcal{X}_{a}$, i.e. $F(\chi)=\langle\chi, \mathbf{f}\rangle$, where the bilinear form $\left\langle\chi, \chi^{*}\right\rangle: \mathcal{X} \times \mathcal{X}^{*} \rightarrow \mathbb{R}$ puts $\mathcal{X}$ and $\mathcal{X}^{*}$ in duality. Together, $\Pi(\chi)=W(\mathbf{D} \chi)-F(\chi)$ is called the total potential and the minimum potential energy principle leads to the general variational problem (2). From the point view of linguistics, if we consider $F(\chi)$ as a subject, $W(\mathbf{w})$ as an object, and the operation "-" as a predicate, then $\Pi(\chi)$ forms a grammatically correct sentence. ${ }^{2}$

According to the action-reaction duality in physics, if there is no action (i.e. $\mathbf{f}=0$ ), the system has non reaction (i.e. $\chi=0$ ). Dually, for any given non-trivial input a real-world problem should have at least one non-trivial solution.

Definition 2 (Properly and Well-Posed Problems) A problem is called properly posed if for any given nontrivial input it has at least one non-trivial solution. It is called well-posed if the solution is unique.

Clearly, this definition is more general than Hadamard's well-posed problems in dynamic systems since the continuity condition is not included. Physically speaking, any real-world problems should be well-posed since all natural phenomena exist uniquely. But practically, it is difficult to model a real-world problem precisely. Therefore, properly posed problems are allowed for the canonical duality theory. This definition is important for understanding the triality theory and NP-hard problems.

For convex stored energy $W: W_{a} \rightarrow \mathbb{R}$, its Legendre conjugate $W^{*}(\sigma)=\{\langle\mathbf{w} ; \boldsymbol{\sigma}\rangle-W(\mathbf{w}) \mid \boldsymbol{\sigma}=\partial W(\mathbf{w})\}$ is uniquely defined on $\mathcal{W}_{a}^{*}$. Thus, by $W(\mathbf{D} \chi)=\langle\mathbf{D} \chi ; \sigma\rangle-W^{*}(\boldsymbol{\sigma})$ the total potential $\Pi(\chi)$ can be written in the Lagrangian form ${ }^{3} L: \mathcal{X}_{a} \times \mathcal{W}_{a}^{*} \rightarrow \mathbb{R}$

$$
L(\chi, \sigma)=\langle\mathbf{D} \chi ; \sigma\rangle-W^{*}(\sigma)-\langle\chi, \mathbf{f}\rangle=\left\langle\chi, \mathbf{D}^{*} \sigma-\mathbf{f}\right\rangle-W^{*}(\sigma)
$$

where $\chi \in \mathcal{X}_{a}$ can be viewed as a Lagrange multiplier for the equilibrium equation $\mathbf{D}^{*} \sigma=\mathbf{f} \in \mathcal{X}_{a}^{*}$. In linear elasticity, $L(\chi, \sigma)$ is the well-known Hellinger-Reissner complementary energy [2]. Let $\mathcal{S}_{c}=\left\{\boldsymbol{\sigma} \in \mathcal{W}_{a}^{*} \mid \mathbf{D}^{*} \sigma=\mathbf{f}\right\}$ be the so-called statically admissible space. Then the Lagrangian dual of the general problem $\left(\mathcal{P}_{0}\right)$ is given by

$$
\left(\mathcal{P}_{0}^{*}\right): \quad \max \left\{\Pi^{*}(\sigma)=-W^{*}(\sigma) \mid \sigma \in \mathcal{S}_{c}\right\}
$$

and the saddle Lagrangian leads to a well-known min-max duality in convex (static) systems:

$$
\min _{\mathcal{X} \in \mathcal{X}_{c}} \Pi(\chi)=\min _{\mathcal{X} \in \mathcal{X}_{a}} \max _{\sigma \in \mathcal{W}_{a}^{*}} L(\chi, \sigma)=\max _{\sigma \in \mathcal{W}_{a}^{*}} \min _{\mathcal{X} \in \mathcal{X}_{a}} L(\chi, \sigma)=\max _{\sigma \in \mathcal{S}_{c}} \Pi^{*}(\sigma) .
$$

\footnotetext{
${ }^{2}$ By the facts that (object, subject) is a duality pair in a noun (or pronoun) space, which is dual to a verb space, the multi-level duality pattern $\{($ object, subject $)$; predicate $\}$ is called triality, which is essential for complicated languages.

${ }^{3}$ In Physics literature, the same notation $L$ is used for both action $L(\chi, \dot{\chi})$ and the Lagrangian $L(\chi, \mathbf{p})$ since both represent the same physical quantity.
} 
This one-to-one duality is called the mono-duality in [2], which holds only for convex problems.

In real-world problems the stored energy $W(\mathbf{w})$ is usually nonconvex in order to model complex phenomena. Its complementary energy can't be determined uniquely by the Legendre transformation. Although its Fenchel conjugate $W^{\sharp}: W_{a}^{*} \rightarrow \mathbb{R} \cup\{+\infty\}$ can be uniquely defined, the Fenchel-Moreau dual problem

$$
\left(\mathcal{P}_{0}^{\sharp}\right): \quad \max \left\{\Pi^{\sharp}(\sigma)=-W^{\sharp}(\sigma) \mid \sigma \in \mathcal{S}_{c}\right\}
$$

is not considered as a complementary-dual problem due to Fenchel-Young inequality: $\theta=\min \Pi(\chi)-\max \Pi^{\sharp}(\sigma) \geq 0$ and $\theta \neq 0$ is the well-known duality gap. This duality gap is intrinsic to all Lagrange-Fenchel-Moreau types duality problems since the linear operator $\mathbf{D}$ can't change the nonconvexity of $W(\mathbf{D} \chi)$. It turns out that the existence of a perfect dual principle was an open problem in both nonlinear analysis and global optimization. The canonical duality theory [2] solved this open problem.

\section{UNIFIED PROBLEM AND CANONICAL DUALITY-TRIALITY THEORY}

Although the objectivity is necessary for real-world modeling, the numerical discretization of $W(\mathbf{D} \chi)$ could lead to a complicated function $W(\mathbf{D} \chi)$, which may not be objective in $\mathbf{w}=\mathbf{D} \chi$. Also in operations research, many challenging problems are artificially proposed. Thus, the objectivity required in Gao and Strang's work on nonlinear elasticity has been relaxed by the canonical duality since 2000 [6].

\section{Canonical Transformation and Gap Function}

In the canonical duality theory, a real-valued function $\Phi: \mathcal{E}_{a} \subset \mathcal{E} \rightarrow \mathbb{R}$ is said to be canonical if the duality relation $\boldsymbol{\xi}=\partial \Phi(\xi): \mathcal{E}_{a} \rightarrow \mathcal{E}_{a}^{*} \subset \mathcal{E}^{*}$ is bijective. The canonical duality is a fundamental principle in sciences and oriental philosophy, which underlies all natural phenomena. Therefore, instead of the objectivity in continuum physics, a generalized objective function $W(\mathbf{w})$ is used in the canonical duality theory under the following assumption.

Assumption $1 \quad$ For a given $W: \mathcal{W}_{a} \rightarrow \mathbb{R}$, there exists a canonical measure $\boldsymbol{\xi}: \mathcal{W}_{a} \rightarrow \mathcal{E}_{a}$ and a canonical function $\Phi: \mathcal{E}_{a} \rightarrow \mathbb{R}$ such that the following conditions hold:

(A1.1) Positivity: $W(\mathbf{w}) \geq 0 \quad \forall \mathbf{w} \in \mathcal{W}_{a}$;

(A1.2) Canonicality: $W(\mathbf{w})=\Phi(\boldsymbol{\xi}(\mathbf{w})) \forall \mathbf{w} \in \mathcal{W}_{a} ;$ And either

(A1.3) Coercivity: $\lim W(\mathbf{w})=\infty$ as $\|\mathbf{w}\| \rightarrow \infty$, or

(A1.3*) Boundness: $\mathcal{W}_{a}$ is bounded.

Generally speaking, (A1.1) and (A1.2) are necessary for any real-world problems; while (A1.3) and (A1.3*) depend mainly on the magnitude of the input $\mathbf{f} \in \mathcal{X}_{a}^{*}$. Usually, the coercivity is for small $\|\mathbf{f}\|$ (within the system's capacity, such as elasticity) and the boundness is for big $\|\mathbf{f}\|$ (beyond the system's capacity, such as plasticity) [5].

Let $\Lambda=\boldsymbol{\xi} \circ \mathbf{D}: \mathcal{X}_{a} \rightarrow \mathcal{E}_{a}$ be the so-called geometrically admissible operator. The canonicality $W(\mathbf{D} \chi)=\Phi(\Lambda(\chi))$ is also called the canonical transformation in the canonical duality theory. Let $\langle\xi ; \boldsymbol{s}\rangle: \mathcal{E} \times \mathcal{E}^{*} \rightarrow \mathbb{R}$ be the bilinear form which puts $\mathcal{E}$ and $\mathcal{E}^{*}$ in duality. By (A1.2), we have $\mathcal{X}_{c}=\left\{\chi \in \mathcal{X}_{a} \mid \Lambda(\chi) \in \mathcal{E}_{a}\right\}$ and the problem $\left(\mathcal{P}_{0}\right)$ can be equivalently reformulated in the following canonical form

$$
(\mathscr{P}): \min \left\{\Pi(\chi)=\Phi(\Lambda(\chi))-\langle\chi, \mathbf{f}\rangle \mid \chi \in \mathcal{X}_{c}\right\} .
$$

By the facts that the canonical duality is a universal principle in nature, the problem $(\mathcal{P})$ can be used to model general complex systems. The criticality condition leads to the following canonical equilibrium equation:

$$
\mathbf{A}(\chi)=\Lambda_{t}^{*}(\chi) \mathbf{C}(\Lambda(\chi))=\mathbf{f},
$$

where $\Lambda_{t}(\chi)=\partial \Lambda(\chi)$ represents a generalized Gâteau (or directional) derivative of $\Lambda(\chi)$, its adjoint $\Lambda_{t}^{*}$ is called the balance operator, $\boldsymbol{\xi}=\mathbf{C}(\boldsymbol{\xi})=\partial \Phi(\boldsymbol{\xi})$ and $\mathbf{C}: \mathcal{E}_{a} \rightarrow \mathcal{E}_{a}^{*}$ is a canonical dual (or constitutive) operator. The canonical equilibrium equation (9) shows a symmetry broken in the canonical equilibrium equation, i.e., instead of $\Lambda^{*}$, the balance operator $\Lambda_{t}^{*}$ is adjoined with $\Lambda_{t}$. It was discovered by Gao and Strang that by introducing a complementary operator $\Lambda_{c}(\chi)=\Lambda(\chi)-\Lambda_{t}(\chi) \chi$, this locally broken symmetry is recovered by a so-called complementary gap function

$$
G_{a p}(\chi, \boldsymbol{s})=\left\langle-\Lambda_{c}(\chi) ; \boldsymbol{s}\right\rangle
$$

which plays a key role in global optimization and the triality theory. 


\section{Complementary-Dual Principle and Analytical Solution}

By the Legendre transforation, the conjugate $\Phi^{*}(\boldsymbol{s})=\left\{\langle\boldsymbol{\xi} ; \boldsymbol{s}\rangle-\Phi(\boldsymbol{\xi}) \mid \boldsymbol{s}=\partial \Phi(\boldsymbol{\xi}) \quad \forall \boldsymbol{\xi} \in \mathcal{E}_{a}\right\}$ can be uniquely defined and the following canonical duality relations hold on $\mathcal{E}_{a} \times \mathcal{E}_{a}^{*}$ :

$$
\boldsymbol{\varsigma}=\partial \Phi(\boldsymbol{\varepsilon}) \Leftrightarrow \boldsymbol{\varepsilon}=\partial \Phi^{*}(\boldsymbol{\zeta}) \Leftrightarrow \Phi(\boldsymbol{\varepsilon})+\Phi^{*}(\boldsymbol{\zeta})=\langle\boldsymbol{\varepsilon} ; \boldsymbol{\zeta}\rangle .
$$

If the canonical function is convex and lower semi-continuous, the Gâteau derivative $\partial$ should be replaced by the sub-differential and $\Phi^{*}$ is replaced by the Fenchel conjugate $\Phi^{\sharp}(\boldsymbol{\zeta})=\sup \left\{\langle\boldsymbol{\xi} ; \boldsymbol{s}\rangle-\Phi(\boldsymbol{\xi}) \mid \boldsymbol{\xi} \in \mathcal{E}_{a}\right\}$. In this case, (11) is replaced by the generalized canonical duality

$$
\boldsymbol{s} \in \partial \Phi(\boldsymbol{\varepsilon}) \Leftrightarrow \boldsymbol{\varepsilon} \in \partial \Phi^{\sharp}(\boldsymbol{\zeta}) \Leftrightarrow \Phi(\boldsymbol{\varepsilon})+\Phi^{\sharp}(\boldsymbol{s})=\langle\boldsymbol{\varepsilon} ; \boldsymbol{s}\rangle \forall(\boldsymbol{\xi}, \boldsymbol{s}) \in \mathcal{E}_{a} \times \mathcal{E}_{a}^{*} .
$$

If the convex set $\mathcal{E}_{a}$ contains inequality constrains, then (12) includes all the internal KKT conditions [14, 5]. In this sense, a KKT point of the canonical form $\Pi(\chi)$ is a generalized critical point of $\Pi(\chi)$.

By the complementarity $\Phi(\Lambda(\chi))=\langle\Lambda(\chi) ; \boldsymbol{s}\rangle-\Phi^{*}(\boldsymbol{s})$, the canonical form of $\Pi(\chi)$ can be equivalently written in Gao and Strang's total complementary function $\Xi: \mathcal{X}_{a} \times \mathcal{E}_{a}^{*} \rightarrow \mathbb{R}[3]$

$$
\Xi(\chi, \boldsymbol{s})=\langle\Lambda(\chi) ; \boldsymbol{s}\rangle-\Phi^{*}(\boldsymbol{s})-\langle\chi, \mathbf{f}\rangle .
$$

Then, the canonical dual function can be obtained by the canonical dual transformation:

$$
\Pi^{d}(\chi)=\operatorname{sta}\left\{\Xi(\chi, \boldsymbol{s}) \mid \chi \chi \mathcal{X}_{a}\right\}=G_{a p}^{\Lambda}(\boldsymbol{\varsigma})-\Phi^{*}(\boldsymbol{\varsigma})
$$

where $\operatorname{sta}\{f(\chi) \mid \chi \in \mathcal{X}\}$ stands for finding the stationary value of $f(\chi)$ on $\mathcal{X}, G_{a p}^{\Lambda}(\boldsymbol{s})=\operatorname{sta}\left\{\langle\Lambda(\chi) ; \boldsymbol{s}\rangle-\langle\chi, \mathbf{f}\rangle \mid \chi \in \mathcal{X}_{a}\right\}$, which is defined on the canonical dual feasible space $\mathcal{S}_{c}=\left\{\boldsymbol{s} \in \mathcal{E}_{a}^{*} \mid \Lambda_{t}^{*}(\chi) \boldsymbol{s}=\mathbf{f} \forall \chi \in \mathcal{X}_{a}\right\}$. Clearly, $\mathcal{S}_{c} \neq \emptyset$ if $(\mathcal{P})$ is properly posed.

Theorem 1 (Complementary-Dual Principle [2]) The pair $(\overline{\boldsymbol{\chi}}, \overline{\boldsymbol{\zeta}})$ is a critical point of $\Xi(\chi, \boldsymbol{\varsigma})$ if and only if $\bar{\chi}$ is a critical point of $\Pi(\chi)$ and $\overline{\boldsymbol{\zeta}}$ is a critical point of $\Pi^{d}(\boldsymbol{\varsigma})$. Moreover,

$$
\Pi(\bar{\chi})=\Xi(\bar{\chi}, \overline{\boldsymbol{\zeta}})=\Pi^{d}(\overline{\boldsymbol{\zeta}}) .
$$

Proof. The criticality condition $\partial \Xi(\bar{\chi}, \overline{\boldsymbol{\zeta}})=0$ leads to the following canonical equations

$$
\Lambda(\bar{\chi})=\partial \Phi^{*}(\overline{\boldsymbol{\zeta}}), \quad \Lambda_{t}^{*}(\bar{\chi}) \overline{\boldsymbol{\zeta}}=\mathbf{f} .
$$

The theorem is proved by the canonical duality (11) and the definition of $\Pi^{d}$.

Theorem 1 shows a one-to-one correspondence of the critical points between the primal function and its canonical dual. This theorem solved the fifty-year old open problem on complementary variational principle in nonlinear mechanics and is known as the Gao principle in literature [17]. In real-world applications, the geometrical operator $\Lambda$ is usually quadratic homogeneous i.e. $\Lambda(\alpha \chi)=\alpha^{2} \Lambda(\chi) \quad \forall \alpha \in \mathbb{R}$. In this case, we have [3] $\Lambda_{t}(\chi) \chi=2 \Lambda(\chi), \quad \Lambda_{c}(\chi)=-\Lambda(\chi)$ and

$$
\Xi(\chi, \boldsymbol{s})=G_{a p}(\chi, \boldsymbol{s})-\Phi^{*}(\boldsymbol{s})-\langle\chi, \mathbf{f}\rangle=\frac{1}{2}\langle\chi, \mathbf{G}(\boldsymbol{s}) \chi\rangle-\Phi^{*}(\boldsymbol{s})-\langle\chi, \mathbf{f}\rangle,
$$

where $\mathbf{G}(\boldsymbol{s})=\partial_{\chi}^{2} G_{a p}(\chi, \boldsymbol{s})$. Then, the canonical dual function $\Pi^{d}(\boldsymbol{s})$ can written explicitly as

$$
\Pi^{d}(\boldsymbol{\varsigma})=\left\{\Xi(\chi, \boldsymbol{s}) \mid \mathbf{G}(\boldsymbol{\varsigma}) \chi=\mathbf{f} \forall \chi \in \mathcal{X}_{a}\right\}=-\frac{1}{2}\left\langle[\mathbf{G}(\boldsymbol{s})]^{+} \mathbf{f}, \mathbf{f}\right\rangle-\Phi^{*}(\boldsymbol{s}),
$$

where $\mathbf{G}^{+}$represents a generalized inverse of $\mathbf{G}$.

$$
\begin{gathered}
\text { Theorem } 2 \text { (Analytical Solution Form[2]) } \quad \text { If } \overline{\boldsymbol{\zeta}} \in \mathcal{S}_{c} \text { is a critical point of } \Pi^{d}(\boldsymbol{S}) \text {, then } \\
\bar{\chi}=[\mathbf{G}(\overline{\boldsymbol{\zeta}})]^{+} \mathbf{f}
\end{gathered}
$$

is a critical point of $\Pi(\chi)$ and $\Pi(\bar{\chi})=\Xi(\bar{\chi}, \overline{\boldsymbol{\zeta}})=\Pi^{d}(\overline{\boldsymbol{\zeta}})$. Dually, if $\bar{\chi} \in \mathcal{X}_{c}$ is a critical point of $\Pi(\chi)$, it must be in the form of (19) for a critical point $\overline{\boldsymbol{\zeta}} \in \mathcal{S}_{c}$ of $\Pi^{d}(\boldsymbol{\varsigma})$.

This unified analytical solution form holds not only for general global optimization problems in finite dimensional systems, but also for a large-class of nonlinear boundary/initial value problems in nonconvex analysis and dynamic systems [12]. 


\section{Triality Theory and NP-Hard Criterion}

Let $\bar{\chi} \in \mathcal{X}_{c}$ be a critical point of a real-valued function $\Pi: \mathcal{X}_{c} \rightarrow \mathbb{R} . \bar{\chi}$ is called degenerate (res. non-degenerate) if the Hessian matrix of $\Pi(\chi)$ is singular (resp. non-singular) at $\bar{\chi}$. The function $\Pi: \mathcal{X}_{c} \rightarrow \mathbb{R}$ is called a Morse function if it has no degenerate critical points.

Theorem 3 (Triality Theory [6]) Suppose that $\Phi: \mathcal{E}_{a} \rightarrow \mathbb{R}$ is convex, $(\bar{\chi}, \overline{\boldsymbol{\zeta}})$ is a non-degenerate critical point of $\Xi(\chi, \boldsymbol{\varsigma})$ and $\mathcal{X}_{o} \times \mathcal{S}_{o}$ is a neighborhood ${ }^{4}$ of $(\overline{\boldsymbol{\chi}}, \overline{\boldsymbol{\zeta}})$. If $\overline{\boldsymbol{\varsigma}} \in \mathcal{S}_{c}^{+}=\left\{\boldsymbol{\varsigma} \in \mathcal{S}_{c} \mid \mathbf{G}(\boldsymbol{\boldsymbol { s }}) \geq 0\right\}$, then

$$
\Pi(\bar{\chi})=\min _{\boldsymbol{\chi} \in \mathcal{X}_{c}} \Pi(\chi)=\max _{\boldsymbol{\zeta} \in \mathcal{S}_{c}^{+}} \Pi^{d}(\boldsymbol{s})=\Pi^{d}(\overline{\boldsymbol{S}}) .
$$

If $\overline{\boldsymbol{S}} \in \mathcal{S}_{c}^{-}=\left\{\boldsymbol{\varsigma} \in \mathcal{S}_{c} \mid \mathbf{G}(\boldsymbol{s}) \prec 0\right\}$, then either

or $($ if $\operatorname{dim} \chi=\operatorname{dim} \boldsymbol{\varsigma})$

$$
\begin{aligned}
& \Pi(\bar{\chi})=\max _{\boldsymbol{\chi} \in \mathcal{X}_{o}} \Pi(\chi)=\max _{\boldsymbol{\zeta} \in \mathcal{S}_{o}} \Pi^{d}(\boldsymbol{\varsigma})=\Pi^{d}(\overline{\boldsymbol{\zeta}}), \\
& \Pi(\bar{\chi})=\min _{\boldsymbol{\chi} \in \mathcal{X}_{o}} \Pi(\chi)=\min _{\boldsymbol{\boldsymbol { \zeta }} \in \mathcal{S}_{o}} \Pi^{d}(\boldsymbol{\varsigma})=\Pi^{d}(\overline{\boldsymbol{\zeta}}) .
\end{aligned}
$$

The statement (20) is the so-called canonical min-max duality, which can be proved easily by Gao and Strang's work in 1989 [3]. Clearly, $\boldsymbol{\varsigma} \in \mathcal{S}_{c}^{+}$if and only if $G_{a p}(\chi, \boldsymbol{\varsigma}) \geq 0 \forall \chi \in \mathcal{X}$. This duality theory shows that the Gao-Strang gap function provides a global optimum criterion. The statements (21) and (22) are called the canonical double-max and double-min dualities, respectively, which can be used to find local extremum solutions.

Theorem 3 shows that the nonconvex problem $(\mathcal{P})$ is canonically dual to a concave maximum stationary problem:

$$
\left(\mathcal{P}^{d}\right): \max \operatorname{sta}\left\{\Pi^{d}(\boldsymbol{\varsigma}) \mid \boldsymbol{\varsigma} \in \mathcal{S}_{c}^{+}\right\}
$$

Theorem 4 (Existence and uniqueness criteria [9]) For a properly posed $(\mathcal{P})$, if the canonical function $\Phi$ : $\mathcal{E}_{a} \rightarrow \mathbb{R}$ is convex, int $\mathcal{S}_{c}^{+} \neq \emptyset$, and

$$
\lim _{\alpha \rightarrow 0^{+}} \Pi^{d}\left(\boldsymbol{S}_{o}+\alpha \boldsymbol{S}\right)=-\infty \quad \forall \boldsymbol{S}_{o} \in \partial \mathcal{S}_{c}^{+}, \forall \boldsymbol{s} \in \mathcal{S}_{c}^{+}
$$

then $\left(\mathcal{P}^{d}\right)$ has at least one solution $\overline{\boldsymbol{\zeta}} \in \mathcal{S}_{c}^{+}$and $\bar{\chi}=[\mathbf{G}(\overline{\boldsymbol{S}})]^{+} \mathbf{f}$ is a solution to $(\mathcal{P})$. The solution is unique if $\partial \mathbf{G}(\overline{\boldsymbol{\zeta}})>0$.

Proof. Under the required conditions $-\Pi^{d}: \mathcal{S}_{c}^{+} \rightarrow \mathbb{R}$ is convex and coercive and int $\mathcal{S}_{c}^{+} \neq \emptyset$. Therefore, $\left(\mathcal{P}^{d}\right)$ has at least one solution. If $\partial \mathbf{G}(\overline{\boldsymbol{S}})>0$, then $\Pi^{d}: \mathcal{S}_{c}^{+} \rightarrow \mathbb{R}$ is strictly concave and $\left(\mathcal{P}^{d}\right)$ has a unique solution.

This theorem shows that if int $\mathcal{S}_{c}^{+} \neq \emptyset$ the nonconvex problem $(\mathcal{P})$ is canonically dual to $\left(\mathcal{P}^{d}\right)$ which can be solved easily. Otherwise, the problem $(\mathscr{P})$ is canonically dual to the following minimal stationary problem, i.e. to find a global minimum stationary value of $\Pi^{d}$ on $\mathcal{S}_{c}$ :

$$
\left(\mathcal{P}^{s}\right): \min \operatorname{sta}\left\{\Pi^{d}(\boldsymbol{\varsigma}) \mid \boldsymbol{\varsigma} \in \mathcal{S}_{c}\right\}
$$

which could be really NP-hard since $\Pi^{d}(\boldsymbol{\varsigma})$ is nonconvex on the nonconvex set $\mathcal{S}_{c}$. Therefore, a conjecture was proposed in [8].

\section{Conjecture 1 (Criterion of NP-Hardness) A properly posed problem $(\mathcal{P})$ is NP-hard if and only if int $\mathcal{S}_{c}^{+}=\emptyset$.}

The canonical duality-triality theory has been successfully used for solving a wide class problems in both global optimization and nonconvex analysis [12].

\section{SYMMETRY, NP-HARDNESS AND PERTURBATION METHODS}

The concept of symmetry is closely related to the duality and, in certain sense, can be viewed as a geometric duality. Mathematically, symmetry means invariance under transformation. By the canonicality, the object $W(\mathbf{w})$ possesses naturally certain symmetry. If the subject $F(\chi)=0$, then $\Pi(\chi)=W\left(\mathbf{D}_{\chi}\right)=\Phi(\Lambda(\chi))$ and $(\mathcal{P})$ should have either a trivial solution or multiple solutions due to the symmetry. In this case $\Pi^{d}(\boldsymbol{\varsigma})=-\Phi^{*}(\boldsymbol{\varsigma})$ is concave and, by the triality theory, its critical point $\overline{\boldsymbol{\zeta}} \in \mathcal{S}_{c}^{-}$is a global maximizer, $\bar{\chi}=[\mathbf{G}(\overline{\boldsymbol{\zeta}})]^{+} \mathbf{f}=0$ is the biggest local maximizer of $\Pi(\chi)$, while

\footnotetext{
${ }^{4}$ The neighborhood $\mathcal{X}_{o}$ of $\bar{\chi}$ means that on which, $\bar{\chi}$ is the only critical point (see page 140 [2]).
} 
the global minimizers $\bar{\chi}(\overline{\boldsymbol{\zeta}})$ must be for those $\overline{\boldsymbol{\zeta}} \in \partial \mathcal{S}_{c}^{+}$such that $\Pi^{d}(\overline{\boldsymbol{\zeta}})=\min \left\{-\Phi^{*}(\boldsymbol{\boldsymbol { S }}) \mid \operatorname{det} \mathbf{G}(\boldsymbol{\boldsymbol { \zeta }})=0 \quad \forall \boldsymbol{\varsigma} \in \mathcal{S}_{c}\right\}$. Clearly, this nonconvex constrained concave minimization problem could be really NP-hard. Therefore, many wellknown NP-hard problems in computer science and global optimization are not well-posed problems. Such as the maxcut problem, which is a special case of quadratic integer programming problem $\left(\mathcal{P}_{q i}\right)$. Due to the symmetry $\mathbf{Q}=\mathbf{Q}^{T}$ and $\mathbf{f}=0$, its canonical dual problem has multiple solutions on the boundary of $\mathcal{S}_{c}^{+}$. The problem is considered as NPcomplete even if $Q_{i j}=1$ for all edges. Strictly speaking, this is not a real-world problem but only a geometrical model. Without sufficient geometrical constraints in $\mathcal{X}_{a}$, the graph is not physically fixed and any rigid motion is possible. However, by adding a linear perturbation $\mathbf{f} \neq 0$, this problem can be solved efficiently by the canonical duality theory [20]. Also it was proved by the author $[9,11]$ that the general quadratic integer problem $\left(\mathcal{P}_{q i}\right)$ has a unique solution as long as the input $\mathbf{f} \neq 0$ is big enough. These results show that the subjective function plays an essential role for symmetry breaking to leads a well-posed problem.

Conjecture 2 For any given properly posed problem $(\mathcal{P})$ under the Assumption 1, there exists a $f_{c}>0$ such that $\left(\mathcal{P}^{d}\right)$ has a unique solution in $\mathcal{S}_{c}^{+}$as long as $\|\mathbf{f}\| \geq f_{0}$.

This conjecture shows that any properly posed problems are not NP-hard if the input $\|\mathbf{f}\|$ is big enough. Generally speaking, most NP-hard problems have multiple solutions located either on the boundary or the outside of $\mathcal{S}_{c}^{+}$. Therefore, a quadratic perturbation method can be suggested as

$$
\Xi_{\delta_{k}}(\boldsymbol{\chi}, \boldsymbol{s})=\Xi(\chi, \boldsymbol{s})+\frac{1}{2} \delta_{k}\left\|\chi-\chi_{k}\right\|^{2}=\frac{1}{2}\left\langle\chi, \mathbf{G}_{\delta_{k}}(\boldsymbol{s}) \chi\right\rangle-\Phi^{*}(\boldsymbol{s})-\left\langle\chi, \mathbf{f}_{\delta_{k}}\right\rangle+\frac{1}{2} \delta_{k}\left\langle\chi_{k}, \chi_{k}\right\rangle,
$$

where $\delta_{k}>0, \boldsymbol{\chi}_{k}(k=1,2, \ldots)$ are perturbation parameters, $\mathbf{G}_{\delta_{k}}(\boldsymbol{\zeta})=\mathbf{G}(\boldsymbol{\zeta})+\delta_{k} \mathbf{I}$, and $\mathbf{f}_{\delta_{k}}=\mathbf{f}+\delta_{k} \boldsymbol{\chi}_{k}$. Thus, the original canonical dual feasible space $\mathcal{S}_{c}^{+}$can be enlarged to $\mathcal{S}_{\delta_{k}}^{+}=\left\{\boldsymbol{s} \in \mathcal{S}_{c} \mid \mathbf{G}_{\delta_{k}}(\boldsymbol{S})>0\right\}$. Thus, a perturbed canonical dual problem can be proposed as

$$
\left(\mathcal{P}_{k}^{d}\right): \max \left\{\min \left\{\Xi_{\delta_{k}}(\chi, \boldsymbol{s}) \mid \chi \in \mathcal{X}_{a}\right\} \mid \boldsymbol{s} \in \mathcal{S}_{\delta_{k}}^{+}\right\}
$$

and a canonical primal-dual algorithm has been developed with successful applications for solving sensor network optimization problems [19] and chaotic dynamics [15].

\section{RELATION WITH SDP PROGRAMMING}

Now let us show the relation between the canonical duality theory and the semi-definite programming relaxation.

Theorem $5 \quad$ Suppose that $\Phi: \mathcal{E}_{s} \rightarrow \mathbb{R}$ is convex and $\overline{\boldsymbol{s}} \in \mathcal{E}_{a}^{*}$ is a solution of the problem

$$
\left(\mathcal{P}^{s d}\right): \min \left\{g+\Phi^{*}(\boldsymbol{\zeta})\right\} \quad \text { s.t. }\left(\begin{array}{cc}
\mathbf{G}(\boldsymbol{\zeta}) & \mathbf{f} \\
\mathbf{f}^{T} & 2 g
\end{array}\right) \geq 0 \quad \forall \boldsymbol{\varsigma} \in \mathcal{E}_{a}^{*}, \quad g \in \mathbb{R},
$$

then $\boldsymbol{\chi}=[\mathbf{G}(\boldsymbol{S})]^{+} \mathbf{f}$ is a global minimum solution to the nonconvex problem $(\mathcal{P})$.

Proof. The problem $\left(\mathcal{P}^{d}\right)$ can be equivalently written in the following problem

$$
\min \left\{g+\Phi^{*}(\boldsymbol{s}) \mid g \geq G_{a p}^{\Lambda}(\boldsymbol{s}), \mathbf{G}(\boldsymbol{s}) \geq 0 \quad \forall \boldsymbol{s} \in \mathcal{E}_{a}^{*}\right\} .
$$

Then, by using the Schur complement Lemma, this problem is equivalent to $\left(\mathcal{P}^{s d}\right)$. The theorem is proved by the triality theory.

It was proved [11] that for the same problem $\left(\mathcal{P}_{q i}\right)$, if we use different geometrical operator

$$
\Lambda(\mathbf{x})=\mathbf{x} \mathbf{x}^{T} \in \mathcal{E}_{a}=\left\{\boldsymbol{\xi} \in \mathbb{R}^{n \times n} \mid \boldsymbol{\xi}=\boldsymbol{\xi}^{T}, \quad \boldsymbol{\xi} \geq 0, \operatorname{rank} \boldsymbol{\xi}=1, \quad \xi_{i i}=1 \quad \forall i=1, \ldots, n\right\},
$$

and the associated canonical function $\Phi(\boldsymbol{\xi})=\frac{1}{2}\langle\boldsymbol{\xi} ; \mathbf{Q}\rangle+\left\{0\right.$ if $\boldsymbol{\xi} \in \mathcal{E}_{a},+\infty$ otherwise $\}$, where $\langle\boldsymbol{\xi}$; $\boldsymbol{\boldsymbol { s }}\rangle=\operatorname{tr}\left(\boldsymbol{\xi}^{T} \boldsymbol{\boldsymbol { s }}\right)$, we should obtain the same canonical dual problem $\left(\mathcal{P}_{q i}^{d}\right)$. Particularly, if $\mathbf{f}=0$, then $\left(\mathcal{P}_{q i}\right)$ is a typical linear semi-definite programming $\min \frac{1}{2}\langle\boldsymbol{\xi} ; \mathbf{Q}\rangle$ s.t. $\boldsymbol{\xi} \in \mathcal{E}_{a}$. Since $\mathcal{E}_{a}$ is not bounded and there is no input, this problem is not properly posed, which could have either no solution or multiple solutions for a given indefinite $\mathbf{Q}=\mathbf{Q}^{T}$. 


\section{APPLICATIONS IN COMPLEX SYSTEMS}

\section{Example 1. Unconstrained Nonconvex Optimization Problem}

$$
(\mathcal{P}): \min \left\{\Pi(\chi)=\sum_{s=1}^{m} \Phi_{s}\left(\Lambda_{s}(\chi)\right)-\langle\chi, \mathbf{f}\rangle \mid \chi \in \mathcal{X}_{c}\right\}
$$

where the canonical measures $\boldsymbol{\xi}_{s}=\Lambda_{s}(\chi)$ could be either a scalar or a tensor, $\Phi_{k}\left(\boldsymbol{\xi}_{k}\right)$ are any given canonical functions, such as polynomial, exponential, logarithm, and their compositions, etc. For example, if $\chi \in \mathcal{X}_{c} \subset \mathbb{R}^{n}$ and

$$
W(\mathbf{D} \boldsymbol{\chi})=\sum_{i \in \mathbb{I}} \frac{1}{2} \alpha_{i} \boldsymbol{\chi}^{T} \mathbf{Q}_{i} \chi+\sum_{j \in \mathbb{J}} \frac{1}{2} \alpha_{j}\left(\frac{1}{2} \chi^{T} \mathbf{Q}_{j} \boldsymbol{\chi}+\beta_{j}\right)^{2}+\sum_{k \in \mathbb{K}} \alpha_{k} \exp \left(\frac{1}{2} \chi^{T} \mathbf{Q}_{k} \boldsymbol{\chi}\right)+\sum_{\ell \in \mathbb{L}} \frac{1}{2} \alpha_{\ell} \boldsymbol{\chi}^{T} \mathbf{Q}_{\ell} \boldsymbol{\chi} \log \left(\frac{1}{2} \chi^{T} \mathbf{Q}_{\ell} \boldsymbol{\chi}\right)
$$

where $\left\{\mathbf{Q}_{s}\right\}$ are positive-definite matrices to allow the Cholesky decomposition $\mathbf{Q}_{s}=\mathbf{D}_{s}^{T} \mathbf{D}_{s}$ for all $s \in\{\mathbb{I}, \mathbb{J}, \mathbb{K}, \mathbb{L}\}$ and $\left\{\alpha_{s}, \beta_{s}\right\}$ are physical constants, which could be either positive or negative under Assumption 1. This general function includes naturally the so-called d.c. functions (i.e. difference of convex functions). By using the canonical measure

$$
\boldsymbol{\xi}=\left\{\xi_{s}\right\}=\left\{\frac{1}{2} \alpha_{i} \chi^{T} \mathbf{Q}_{i} \chi, \frac{1}{2} \chi^{T} \mathbf{Q}_{r} \chi\right\} \in \mathcal{E}_{a}=\mathbb{R}^{p} \times \mathbb{R}_{+}^{q}, \quad p=\operatorname{dim} \mathbb{I}, \quad q=\operatorname{dim} \mathbb{J}+\operatorname{dim} \mathbb{K}+\operatorname{dim} \mathbb{L}
$$

where $\mathbb{R}_{+}^{q}=\left\{\mathbf{x} \in \mathbb{R}^{q} \mid x_{i} \geq 0 \forall i=1, \ldots, q\right\}, W(\mathbf{w})$ can be written in the canonical form

$$
\Phi(\boldsymbol{\xi})=\sum_{i \in \mathbb{I}} \xi_{i}+\sum_{j \in \mathbb{J}} \frac{1}{2} \alpha_{j}\left(\xi_{j}+\beta_{j}\right)^{2}+\sum_{k \in \mathbb{K}} \alpha_{k} \exp \xi_{k}+\sum_{\ell \in \mathbb{L}} \alpha_{\ell} \xi_{\ell} \log \xi_{\ell} .
$$

Thus, $\partial \Phi(\xi)=\left\{1, \varsigma_{r}\right\}$ in which, $\boldsymbol{\varsigma}=\left\{\alpha_{j}\left(\xi_{j}+\beta_{j}\right), \alpha_{k} \exp \xi_{k}, \alpha_{\ell}\left(\log \xi_{\ell}-1\right)\right\} \in \mathcal{E}_{a}^{*}$ and

$$
\mathcal{E}_{a}^{*}=\left\{\boldsymbol{\varsigma} \in \mathbb{R}^{q} \mid \boldsymbol{\varsigma}_{j} \geq-\alpha_{j} \beta_{j} \quad \forall j \in \mathbb{J}, \quad \boldsymbol{\varsigma}_{k} \geq \alpha_{k} \quad \forall k \in \mathbb{K}, \quad \boldsymbol{\zeta}_{\ell} \in \mathbb{R} \quad \forall \ell \in \mathbb{L}\right\} .
$$

The conjugate of $\Phi$ can be easily obtained as

$$
\Phi^{*}(\boldsymbol{s})=\sum_{j \in \mathbb{J}}\left(\frac{1}{2 \alpha_{j}} \varsigma_{j}^{2}+\beta_{j} \varsigma_{j}\right)+\sum_{k \in \mathbb{K}} \varsigma_{k}\left(\ln \left(\alpha_{k}^{-1} \varsigma_{k}\right)-1\right)+\sum_{\ell \in \mathbb{L}} \alpha_{\ell} \exp \left(\alpha_{\ell}^{-1} \varsigma_{\ell}-1\right) .
$$

Since $\Lambda(\chi)$ is quadratic homogenous, the gap function and $G_{a p}^{\Lambda}$ in this case are

$$
G_{a p}(\chi, \boldsymbol{s})=\frac{1}{2} \chi^{T} \mathbf{G}(\boldsymbol{s}) \chi, \quad G_{a p}^{\Lambda}(\boldsymbol{s})=\frac{1}{2} \mathbf{f}^{T}[\mathbf{G}(\boldsymbol{s})]^{+} \mathbf{f}, \quad \mathbf{G}(\boldsymbol{s})=\sum_{i \in \mathbb{I}} \alpha_{i} \mathbf{Q}_{i}+\sum_{s \in\{\mathbb{J}, \mathbb{K}, \mathbb{L}\}} \varsigma_{s} \mathbf{Q}_{s} .
$$

Since $\Pi^{d}(\boldsymbol{\zeta})=-G_{a p}^{\Lambda}(\boldsymbol{\zeta})-\Phi^{*}(\boldsymbol{S})$ is concave and $\mathcal{S}_{c}^{+}$is a closed convex set, if for the given physical constants and the input $\mathbf{f}$ such that $\mathcal{S}_{c}^{+} \neq \emptyset$, the canonical dual problem $\left(\mathcal{P}^{d}\right)$ has at least one solution $\overline{\boldsymbol{\zeta}} \in \mathcal{S}_{c}^{+} \subset \mathbb{R}^{q}$ and $\bar{\chi}=[\mathbf{G}(\overline{\boldsymbol{S}})]^{+} \mathbf{f} \in \mathcal{X}_{c} \subset \mathbb{R}^{n}$ is a global minimum solution to $(\mathcal{P})$. If $n \gg q$, the problem $\left(\mathcal{P}^{d}\right)$ can be much easier than $(\mathcal{P})$.

\section{Example 2. Mixed Integer Nonlinear Programming (MINLP)}

The decision variable for (MINLP) is $\chi=\{\mathbf{y}, \mathbf{z}\} \in \mathcal{Y}_{a} \times \mathcal{Z}_{a}$, where $\mathcal{Y}_{a}$ is a continuous variable set and $\mathcal{Z}_{a}$ is a set of integers. It was shown in [18] that for any given integer set $\mathcal{Z}_{a}$, there exists a linear transformation $\mathbf{D}_{z}: \mathcal{Z}_{a} \rightarrow \mathbb{Z}=$ $\{ \pm 1\}^{n}$. Thus, based on the unified model $(\mathcal{P})$, a general (MINLP) problem can be proposed as

$$
\left(\mathcal{P}_{m i}\right): \min \left\{\Pi(\mathbf{y}, \mathbf{z})=W\left(\mathbf{D}_{y} \mathbf{y}, \mathbf{D}_{z} \mathbf{z}\right)-\langle\mathbf{y}, \mathbf{s}\rangle-\langle\mathbf{z}, \mathbf{t}\rangle \mid(\mathbf{y}, \mathbf{z}) \in \mathcal{Y}_{c} \times \mathcal{Z}_{c}\right\}
$$

where $\mathbf{f}=(\mathbf{s}, \mathbf{t})$ is a given input, $\mathbf{D} \boldsymbol{\chi}=\left(\mathbf{D}_{y} \mathbf{y}, \mathbf{D}_{z} \mathbf{z}\right) \in \mathcal{W}_{y} \times \mathbb{Z}$ is a multi-scale operator, and

$$
\boldsymbol{Y}_{c}=\left\{\mathbf{y} \in \mathcal{Y}_{a} \mid \mathbf{D}_{y} \mathbf{y} \in \mathcal{W}_{y}\right\}, \quad \mathcal{Z}_{c}=\left\{\mathbf{z} \in \mathcal{Z}_{a} \mid \mathbf{D}_{z} \mathbf{z} \in \mathbb{Z}\right\}
$$

In $\mathcal{Y}_{a}$ certain linear constraints are given. Since the set $\mathbb{Z}_{a}$ is bounded, by Assumption 1 either $W: \mathcal{W}_{y} \rightarrow \mathbb{R}$ is coercive or $\mathcal{W}_{y}$ is bounded. This general problem $\left(\mathcal{P}_{m i}\right)$ covers many real-world applications, including the so-called fixed cost problem [13]. Let

$$
\boldsymbol{\epsilon}=\boldsymbol{\Lambda}_{z}(\mathbf{z})=\left(\mathbf{D}_{z} \mathbf{z}\right) \circ\left(\mathbf{D}_{z} \mathbf{z}\right) \in \mathcal{E}_{z}=\mathbb{R}_{+}^{n}
$$

where $\mathbf{x} \circ \mathbf{y}=\left\{x_{i} y_{i}\right\}^{n}$ is the Hadamard product in $\mathbb{R}^{n}$, the integer constraint in $\mathbb{Z}$ can be relaxed by the canonical function $\Psi(\boldsymbol{\epsilon})=\{0$ if $\boldsymbol{\epsilon} \leq \mathbf{e}, \infty$ otherwise $\}$, where $\mathbf{e}=\{1\}^{n}$. Therefore, the canonical form of $\left(\mathcal{P}_{m i}\right)$ is

$$
\min \left\{\Pi(\mathbf{y}, \mathbf{z})=\Phi(\boldsymbol{\Lambda}(\mathbf{y}, \mathbf{z}))+\Psi\left(\boldsymbol{\Lambda}_{z}(\mathbf{z})\right)-\langle\mathbf{y}, \mathbf{s}\rangle-\langle\mathbf{z}, \mathbf{t}\rangle \mid \mathbf{y} \in \boldsymbol{Y}_{c}\right\}
$$


Since the canonical function $\Psi(\boldsymbol{\epsilon})$ is convex, semi-continuous, its Fenchel conjugate is

$$
\Psi^{\sharp}(\sigma)=\sup \left\{\langle\boldsymbol{\epsilon} ; \sigma\rangle-\Psi(\boldsymbol{\epsilon}) \mid \boldsymbol{\epsilon} \in \mathbb{R}^{n}\right\}=\{\langle\mathbf{e} ; \sigma\rangle \text { if } \sigma \geq 0, \infty \text { otherwise }\} .
$$

The generalized canonical duality relations (12) are $\sigma \geq 0 \Leftrightarrow \boldsymbol{\epsilon} \leq \mathbf{e} \Leftrightarrow\langle\boldsymbol{\epsilon}-\mathbf{e} ; \boldsymbol{\sigma}\rangle=0$. The complementarity shows that the canonical integer constraint $\boldsymbol{\epsilon}=\mathbf{e}$ can be relaxed by the $\sigma>\mathbf{0}$ in continuous space. Thus, if $\boldsymbol{\xi}=\boldsymbol{\Lambda}(\chi)$ is a quadratic homogenous operator and the canonical function $\Phi(\xi)$ is convex on $\mathcal{E}_{a}$, the canonical dual to $\left(\mathcal{P}_{m i}\right)$ is

$$
\left(\mathcal{P}_{m i}^{d}\right): \max \left\{\Pi^{d}(\boldsymbol{s}, \boldsymbol{\sigma})=-\frac{1}{2}\left\langle[\mathbf{G}(\boldsymbol{s}, \boldsymbol{\sigma})]^{+} \mathbf{f}, \mathbf{f}\right\rangle-\Phi^{\sharp}(\boldsymbol{s})-\langle\mathbf{e} ; \boldsymbol{\sigma}\rangle \mid(\boldsymbol{s}, \boldsymbol{\sigma}) \in \mathcal{S}_{c}^{+}\right\},
$$

where $\mathbf{G}(\boldsymbol{s}, \boldsymbol{\sigma})$ depends on the quadratic operators $\boldsymbol{\Lambda}(\boldsymbol{\chi})$ and $\boldsymbol{\Lambda}_{z}(\mathbf{z}), \mathcal{S}_{c}^{+}$is a convex open set

$$
\mathcal{S}_{c}^{+}=\left\{(\boldsymbol{\varsigma}, \boldsymbol{\sigma}) \in \mathcal{E}_{a}^{*} \times \mathbb{R}_{+}^{n} \mid \mathbf{G}(\boldsymbol{s}, \boldsymbol{\sigma}) \geq 0, \quad \boldsymbol{\sigma}>0\right\} .
$$

Applications for general mixed integer programming problems are given in $[11,13]$.

\section{ACKNOWLEDGMENT}

This research is supported by US Air Force Office of Scientific Research (AOARD) under the grant (FA2386-16-14082): A Breakthrough Theory and Algorithm for Solving Chaotic Dynamics and NP-hard Problems in Mathematical Physics and Complex Systems

\section{REFERENCES}

[1] Y. Chen and D.Y. Gao, J. Global Optimization, 64(3), 417-431 (2016).

[2] D.Y. Gao, Duality Principles in Nonconvex Systems: Theory, Methods and Applications (Springer, Boston, 2000).

[3] D.Y. Gao and G. Strang, Quart. Appl. Math. 47(3), 487-504 (1989).

[4] P.G. Ciarlet, Linear and Nonlinear Functional Analysis with Applications, (SIAM, Philadelphia, 2013).

[5] D.Y. Gao, J. Math. Analy. Appl., 221, 672-697 (1998).

[6] D. Y. Gao, J. Glob. Optim. 17(1/4), 127-160 (2000).

[7] D.Y. Gao, Optimization 52(4-5), 467-493 (2003).

[8] D.Y. Gao,J. Indust. Manage. Optim., 3(2), 293-304 (2007).

[9] D.Y. Gao, Comput. E Chem. Eng. 33, 1964-1972 (2009).

[10] D.Y. Gao, On Unified Modeling, Canonical Duality-Triality Theory, Challenges and Breakthrough in Optimization, http://arxiv.org/pdf/1605.05534v3.pdf (2016).

[11] D.Y. Gao and N. Ruan, J. Glob. Optim. 47, 463-484 (2010).

[12] D.Y. Gao, V. Latorre and N. Ruan, Canonical Duality Theory: Unified Methodology for Nonconvex mechanics and Global Optimization, (Spriner, New York 2016).

[13] D.Y. Gao, N. Ruan and H. Sherali, Optimization and Optimal Control, edited by A. Chinchuluun et al. (Springer 2010), pp. 139-156.

[14] V. Latorre and D.Y. Gao, Optimization Letters (2015). http://link.springer.com/article/10.1007/s 11590-0150860-0/fulltext.html

[15] V. Latorre and D.Y. Gao, Int. J. Birfurcation and Chaos, 26, 1650142 (2016). http://arxiv.org/abs/1512.08343

[16] V. Latorre and D.Y. Gao, On half-quadratic reformulation and canonical duality theory in image restoration (2016).

[17] S.F. Li and A. Gupta, J. of Elasticity, 84, 13-31 (2006).

[18] N. Ruan and D.Y. Gao, Global Optimal Solution to Discrete Value Selection Problem with Inequality Constraints (2012). http://arxiv.org/abs/1205.0856

[19] N. Ruan and D.Y. Gao, Performance Evaluations, 75-76, 1-16 (2014).

[20] Z.B. Wang, S.C. Fang, D.Y. Gao and W.X. Xing, J. Glob. Optim., 54, 341-351 (2012). 